\title{
Wenhua Zhao, China CDC's Chief Expert of Nutrition
}

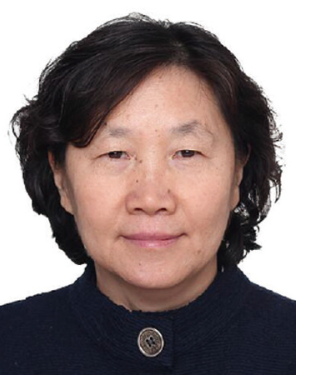

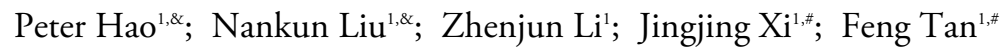

Philippines and later in 2003, a Ph.D. in Nutrition from Kagawa Nutrition University of Japan.

Zhao moved to China CDC (formally known as Chinese Academy of Preventive Medicine, CAPM) in 1996 and since then has made significant contributions to China's public health, particularly in the areas of nutrition epidemiology and dietary assessment methods; national nutrition and NCDs surveillance; obesity and the intervention strategies in China; diabetes and related risk factors; and policy research, health promotion, and education on balanced diet and active lifestyle.

Zhao frequently overcame challenges in adapting international standards to local Chinese settings. For example, differences in food cultures made the Food Frequency Questionnaire (FFQ), a well-established method based on western dietary habits, incapable of being directly applied in China in the 1990s. By 1996, Zhao addressed this problem by leading the establishment of the Chinese Food Frequency Questionnaire (CFFQ) for adults, and the reproducibility and validity of CFFQ was assessed and available by 2000. The CFFQ was successfully applied as the first large-scale epidemiological survey on nutrition and NCDs, which was named the China National Nutritional and Health Survey (CNHS) in 2002 and continued in 2012 and 2015, and was included in the National NCDs Surveillance since 2010.

Zhao has been involved in several national nutrition and NCDs surveillance systems since 2002. For instance, as the Field Director in the 2002 CNHS, she led completion of protocol development, the training of local research teams, quality control, data analysis, project management and coordination, and the completion of the final report issued by The Information Office of the State Council of China.

Based on the 2002 CNHS, Zhao published the first monograph of blood lipid profiles, "Blood Lipid Profile and Prevalence of Dyslipidemia in Chinese Adults". The monograph also promoted the publication of the first edition of Chinese Guidelines for the Management of Dyslipidemia in Adults in 2007.

Zhao has also focused on obesity since 1996, which became one of her key research areas. Her related work provided significant information, such as using waist circumference (WC) as a simpler indicator to predict the risk of central-obesity-related hypertension and diabetes in 2000. For an intervention of childhood obesity, she established the "Nutrition School" pilot program in Shunyi District, Beijing in 2016, and the program subsequently expanded rapidly to 8 provinces by 2017 .

Zhao also focused on a community-based study on maintaining healthy body weight by multisectoral collaboration to promote physical activities for the public. She was the Chief Editor of the Physical Activity Guidelines for Chinese Adults, issued by the Bureau for Disease Control and Prevention of China's Ministry of Health (now the National Health Commission) in 2011. She was also the co-author of a national standard: the Criteria of Weight for Adults in China (WS/T 428-2013).

In 2010, as the Director of National Working Group of the 2010 China Chronic Disease and Behavior Risk Factors Surveillance, Zhao collaborated with Dr. Guang Ning and his team in Shanghai Ruijin Hospital to jointly complete the Diabetes Epidemiology Study, which covered 162 counties in 31 provincial-level administrative divisions (PLADs) and involved almost 100,000 adults in China. This surveillance led to a publication in JAMA in 
2013. It was a remarkable exploration of the collaboration between China CDC and a hospital in the medical system. The research had provided fundamental techniques and experiences for the establishment of the China NCDs Control and Prevention Plan and Healthy China 2030 strategies. Zhao also published the first monograph on the national population distribution of body-mass index (BMI), prevalence of obesity, and 10 years changes among different age groups in China: Present Status of Overweight and Obesity and 10 Years Changes in China.

Zhao also contributed to international public health community. In 2003 and 2004, as a short-term consultant to the World Health Organization (WHO), she provided technical support to the National Food Consumption Survey in Papua New Guinea. She now is a member of WHO and United Nations Children's Fund (UNICEF) Technical Expert Advisory group on nutrition Monitoring (TEAM).

Zhao has extensive experience in policy research, health promotion, and education on balanced diet and active lifestyles nationally and internationally. She has supported national policymaking on NCDs control and prevention, e.g. in the "Health Lifestyle for All" program initiated in 2007 and a joint program by Shandong Province and the Ministry of Health of China on "Salt Reduction" in 2011. In 2020, as the China CDC's Chief Expert of Nutrition, Zhao emphasized the importance of a balanced diet in response to the coronavirus disease 2019 (COVID-19) pandemic in the Press Conference of the Joint Prevention and Control Mechanism of the State Council. Her livestreamed lectures were viewed over 10 million times.

doi: $10.46234 / \mathrm{ccdcw} 2020.207$

\# Corresponding authors: Jingjing Xi, xijj@chinacdc.cn; Feng Tan, tanfeng@chinacdc.cn.

\footnotetext{
${ }^{1}$ Chinese Center for Disease Control and Prevention, Beijing, China.

${ }^{\&}$ Joint first authors.
}

Submitted: September 09, 2020; Accepted: September 16, 2020 